\title{
Harnessing the power of Social Bookmarking for improving tag-based Recommendations
}

\author{
Georgios Pitsilis ${ }^{1}$ and Wei Wang ${ }^{2}$ \\ 1 Computer Science Research, Athens, Greece \\ georgios.pitsilis@gmail.com \\ 2 School of Computer and Information Technology \\ Beijing Jiaotong University, Beijing, China \\ wangwei1@bjtu.edu.cn
}

\begin{abstract}
Social bookmarking and tagging has emerged a new era in user collaboration. Collaborative Tagging allows users to annotate content of their liking, which via the appropriate algorithms can render useful for the provision of product recommendations. It is the case today for tag-based algorithms to work complementary to rating-based recommendation mechanisms to predict the user liking to various products. In this paper we propose an alternative algorithm for computing personalized recommendations of products, that uses exclusively the tags provided by the users. Our approach is based on the idea of using the semantic similarity of the user-provided tags for clustering them into groups of similar meaning. Afterwards, some measurable characteristics of users' Annotation Competency are combined with other metrics, such as user similarity, for computing predictions. The evaluation on data used from a real-world collaborative tagging system, citeUlike, confirmed that our approach outperforms the baseline Vector Space model, as well as other state of the art algorithms [19], predicting the user liking more accurately.
\end{abstract}

Keywords: Recommender Systems, Collaborative Tagging, Affinity Propagation, citeUlike, Taxonomy

\section{Introduction}

Collaborative tagging, a web-based service that is representive of the new Web 2.0 technology, allows users to store and share various kinds of web resources, such as news, blogs, and photos into social data repositories. Resources are stored into self-emerging structures called folksonomies, in the form of a post that combines $a$ ) an identifier of the resource, $b$ ) the user who posted it and $c$ ) a set of tags. Many web-based resource sharing and publishing services, like youtub 3 , flick1 4 , and Amazon 5 have already adopted such model, allowing user-generated

\footnotetext{
3 www.youtube.com

4 www.flickr.com

5 www.amazon.com
} 
tags to facilitate user information search. The concept of using tags for on-line annotation of objects, also known as Social Bookmarking or Collaborative Tagging, constitutes tags as a novel source of information. Although the use of tags has been found very convenient for managing and organizing people's digital material, from the research perspective it seems to have attracted much interest in Recommender Systems (RS) in the recent years, with literature rapidly expanding.

Despite Collaborative Filtering (CF) algorithms being the most adopted techniques for Recommender Systems, the increasing popularity of collaborative tagging systems pushed towards to tags being integrated into the process of recommendation production. Mechanisms which employ the tags alone for computing item recomendations are less common $[8$, while wherever numeric ratings are additionally provided, they are used complementary to tags for computing item recommendations 31. Relying exclusively on the user-provided tags for computing recommendations, it requires that such information is exploited in the best way for achieving satisfactory quality of predictions. This is the case for digital publication services, like flickr, and in general for social networking services, since they provide no-mechanism for numeric ratings-based evaluation of the published content by the users.

Different from numeric ratings, tags also carry sementic information that can be further exploitable. In addition, tag-words can be classified into hierrarchical ordered systems, called taxonomies, structured upon the natual relationships between their elements. Measurements like, Semantic Distance and Relatedness between tags are computable using the taxonomies. Knowing such distance can prove very important when needing to group similar tags together. In some cases grouping can help to overcome issues like polysemy of tags or the use of synonyms by users for annotating the same item. Such issues exist because users behave differently as far as the way they do annotations, expressing their own style on this task, that differs from one user to another.

While exploring the information hidden on tags for improving recommendations has already been a topic for investigation by the research community in the past, the Annotation Competency of users has not been taken into account yet. In this paper we attempt to utilize the power of taxonomies through tag clustering, along with giving a useful insight into the Annotation Competency of the users.

The rest of the paper is organized as follows: In Section 2 we explain our motivation and related work in the field. In Section 3 we reason about the idea of tag clustering in more detail and describe our algorithm. Section 4 is referred to the evaluation tests we performed and the results received, and finally in section 5 we present our conclusions. 


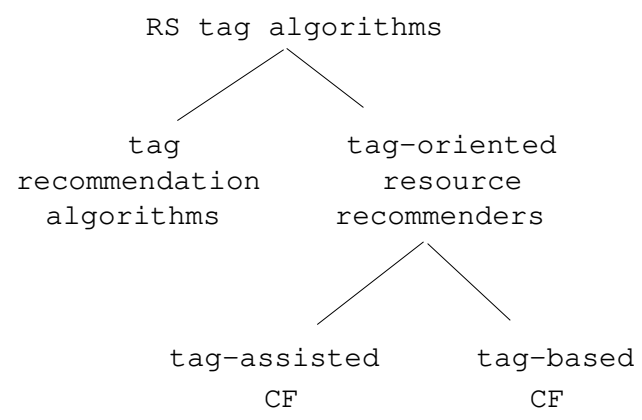

Fig. 1. Taxonomy of algorithms

\section{Related Work and Motivation}

Based on the existing literature, a simple taxonomy of the tag recommender systems could be as depicted in Fig 1 and it is explained as follows: We can distinguish two major types of algorithms, a) tag recommendation algorithms and b) tag-oriented resource recommenders. The first category comprizes solutions aiming to ease the process of annotation by providing personalized recommendations of tags to users about specific items [11 20] 27]. Mechanisms that belong to the first category can either exist as part of a larger concept for resource recommendion, or they can stand as independent services, enabling social network applications to providing automated annotation of various kinds [9] [15]. The tag-oriented category regard prediction models exclusively for resource recommendations, which can be further divided into two sub-categories. For better reference we will call these categories: Tag-assisted CF and Tag-based CF. In tag-assisted $C F$ belong those models which require both item rating values and tags to be available for working out predictions. On the other hand, tag-based $C F$ comprises those models in which the computation of predictions can be performed using the tags alone. The first category has been more explored than the second one, hence the more literature available. It is interesting to note that almost all proposed models that belong to this category perceive the task of recommendation production as a two step process. First, computing the nessesary similarity correlations, and then performing the item predictions using the pre-computing similarities from the first step.

\subsection{Limitations of the Classical Approaches}

There has been a growing number of research efforts that could be classified as tag-assisted CF model. For instance, in 10, 33 21] there has been an effort to user and tag similarities to be combined together into a single expression of similarity, while the work by Parra et.al [16, employs a type of tag-based similarity. Finally, Tso-shutter et.al in [28] introduce a method of fusing userbased with item-based CF, treating the user tags as additional data. 
Nevertheless, we focus our interest on tag-based $C F$ model mainly for two reasons. First, because it is very common for tags to exist as the only available source of information that users provide in a RS, and second, the less constraints imposed by this model extends its suitability to a wider range of applications.

For instance, we will refer to one key model from the literature for tag-based $C F$. In the work by Peng et al. in [19], each tag is viewed as a distinct topic, while the liking of a user to an item is seen as the probability of this user to experience that item. The value of this probability is computed by summing the transition probability through all tags used for tagging this item. The formula they introduced for computing the probability $p(i \mid u)$ that a user $u$ would like item $i$ is given as follows:

$$
p(i \mid u)=\sum_{t \in T} p(t \mid u) \cdot p(i \mid t)
$$

where: $T$ is the set of tags used by user $u$, and $p(t \mid u)$ is the probability that user $u$ uses tag $t$ for item annotation, and $p(i \mid t)$ is the conditional probability of experiencing item $i$ when $\operatorname{tag} t$ is given.

The intuition behind their formula can be phrased as follows: The liking of a user $u$ to item $i$ is highly related to the probability that a particular tag is used by that user, as well as the popularity of this tag when used for annotating item $i$. That probability $p(i \mid u)$ is more or less characteristic to the way that a user makes his own selection of tags for annotating objects. To capture this for a user we introduce the notion of Annotation Competency. We hypothesize that the quality of predictions received by a user would certaintly be affected to some degree by his/her such Competency.

We should also point out that the use of Eq1 becomes inefficient for the reason that users maintain some own collection of words they use for annotating. For that reason it becomes less likely for the probability $p(i \mid u)$ to be computable, thus strongly affecting the avaibalility of predictions. Such formula imposes a serious limitation for the model to work as it requires a significant overlap to exist between the words that various users have used. For the reason that it is very much the case for users' Annotation Competency to be as such, we argue that the above requirement expressed in Eq1 would result to the algorithm performing poorly.

In our opinion, a good model should consider as well any differences that might exist in the Annotation Competency from one user to another.

Another evidence that supports our argument, is the fact that most systems which belong to the above two Tag-oriented categories consider the relationship between the available sources of data as a tripartite structure of items-tags-users. Employing such structure has the weakness of being able to capturing only the two out of the three binany associations at a time, among the tags, entities and users, yet something easy to observe and well mentioned by other researchers in the field 21]. As such, that would result to sparse user-tag and item-tag matrices, which in addition to the above mentioned requirment we set regarding the Annotation Competency would further degrade the recommendation quality. 


\subsection{Solutions for Overcoming the Limitations}

The above remarks suggest the need for taking into account as well the Annotation Competency in the recommendation process. We distinguish the following two central attributes to describe the Annotation Competency of a user.

- The Diversity of Concepts used by a user throughout his/her tagging excercise

- The Annotation Contribution on items.

The attribute of Diversity of Concepts accounts the variety of topics which characterize the profile of a user's interests.

The intuition behind this attribute is driven from the fact that not all users have the same level of experience nor they show the same willingness in their annotation exercise. We consider this attribute to be highly important for the quality of recommendations, mainly because the more experienced or eager users, compared to other users, are meant to be more influencing on the computed predictions. That is because a user who selects tags out from a corpus that includes words from many disciplines, accually provides more data for the system training, and hence he should be regarded to be more contributing than another user whose tags regard only a small area of topics.

As far as the second attribute, Annotation Contribution, while it captures the same requirement of Annotation Competency, as the first one, it though works on item scale. Concerning Annotation Contribution, we perceive that attribute as being expressed by quantitative criteria and it varies from one user to another for a particular item. The quantitative aspect in our case interprets as: The more tags provided for annotating an object the better it is. That is nessesary for distinguishing the contribution of a user who has used few, but identical tags, from another user who has used more descriptive tags for annnotating the same item. For example the tags 'cat' and 'hungry' add more information to the context of the annotated subject than if tags 'cat' and 'hungry' were used. While the first attribute regards the whole reprtoire of tags used by some user in overall, the second one is refered to only some particular annotation experience of that user.

Next we present in more detail our design considerations concerning the two attributes we introduced.

\subsection{Design Considerations concerning the Diversity of Concepts}

To implement the first attibute which expresses the Variety in the Concepts used in a tagging excersize, it requires indentifying all subjects incorprorated into the Annotation Competency of a user.

A straightforard approach to identify the concepts is to partition the tags into distinct Subjects. There are models found in the literature which incorprorate such idea of distincted Subjects. For instance, the work by Peng et al. in [18, follows the concept of organizing the tags into groups. In their work is attempted 
a refinement of the technique proposed by the same authors and is summed in eqn 1 The refined method employs a type of soft clustering called Consistent Nonegative Matrix Factrorization (CNMF), that is a method of applying multivariate analysis onto the tags, for categorizing them into Subjects. Their refined technique is described in the following formula:

$$
p(i \mid u)=\sum_{s \in S} p(s \mid u) \cdot p(i \mid s)
$$

where $p(i \mid u)$ is the predicted liking of user $u$ for item $i, S$ is the set of all identified subjects which tags belong to. $p(s \mid u)$ is the probability of user's $u$ interest in subject $s$ and $p(i \mid s)$ is the probability of experiencing item $i$ when users are interested in subject $s$. Similarly to their model described in eqn, 1, the refined one follows the central intuition of social annotation that is: If user $u$ has used tag $t$ (or a tag of a subject $s$ ) for many times, and tag $t$ (or the subject $s$ ) has been annotated on the article $i$ for many times then it is very likely that user $u$ has strong interest on the article $i$, which should finally be recommended to him/her. From now on, when referring to this formula we will be using the term Topic-Based Variation, for short, while the term Tag-Based Variation will be used when refering to their original method in eq1

A quite similar approach that works on the same idea of distinuishing topics of interests from the tags, has also been proposed by Shepitsen et al. 25]. Their idea works exactly on the same principle expressed in eqn 2 , but it employs hierarchical clustering onto the set of tags for extracting topics. While we consider the solution of partitioning as a very useful one, nevertheless it does not suffciently capture the users' Annotation Competency in the way we expressed it in the two central attributes, for making recommendations. For example, to be in line with the first attribute we set for capturing the diversion in the areas of interest for a user, if applying partitioning alone would not be enough for achieving this.

Furthermore, for the reason that it is quite common for two different people to may have chosen different tags for annotating the same item, it is reasonable to assume that an algorithm that would work on exact matches on the set of tags used, would not be that efficient for identifying similar users. Therefore, the benefit aquired from Collaborative Tagging would not be exploited if using such algorithm.

The logical path to follow would be to group together those tags which are closer to each other, as far as the contextual meaning they carry. From then on in the recommendation proccess, tags would be identified by the cluster ID which they belong to. In this way, it would suffice to simply knowing the user-tocluster associations rather the user-to-item associations, as the existing models require. That would make easier to spot hidden similarities, and any likely common interests between users, which otherwise, due to the sparce user-tag-item relationships, such similarities would not be easily distinguisable. 


\subsection{Design Considerations concerning the Annotation Contribution of a user}

As we mentioned earlier, the second attribute we indruduced requires that Annotation Contribution has also been described using quantitative criteria. Next we will refer to the Vector Space model [24, which is itself a basic tag-based recommendation algorithm, that takes as input the frequencies of tags for computing the distance or similarity between users and items.

We mention Vector Space model here as a good starting point of our consideration as it employs qualitative criteria onto the collection of words used by users for finding potentially interesting items for them.

Roughly, the vector space model works as follows: Each user is represented by a tag vector $u=\left[w_{t_{1}}, w_{t_{2}}, \ldots, w_{t_{n}}\right]$ with $w_{t}$ denoting the weight of the patricular $\operatorname{tag} t$ on that user. Vector weights may be expressed through many ways, with the frequency of tags to be the most common. Likewise, each resource can be modeled as a vector $r=\left[u_{t 1}, u_{t 2}, \ldots, u_{t n}\right]$ over the same set of tags. Next, the user's profiles and the resources can be matched over those tag expressions by computing the similarity value between them. Cosine Similarity can be used to obtain these similarity scores between user profiles and rated resources. Then, by sorting the similairites in decending order we eventually get the $T o p_{\_} N$ list of personalized recommendations of resources for a specific user. The cosine of the two vectors in eqn 3 is derived from the Eucledian dot product formula, with $\left\|\boldsymbol{t}_{\boldsymbol{n}}\right\|$ denote as the length of the vector $t_{n}$, and $\boldsymbol{t}_{\mathbf{1}} \cdot \boldsymbol{t}_{\mathbf{2}}$ is the inner product of the two vectors.

$$
\cos \left(\boldsymbol{t}_{\mathbf{1}}, \boldsymbol{t}_{\mathbf{2}}\right)=\frac{\boldsymbol{t}_{\mathbf{1}} \cdot \boldsymbol{t}_{\mathbf{2}}}{\left\|\boldsymbol{t}_{\mathbf{1}}\right\|\left\|\boldsymbol{t}_{\mathbf{2}}\right\|}
$$

Since frequency values cannot be negative, cosine similarity will range from 0 to 1 , with 1 denoting a perfect match. Adapted versions of the vector space model to work with folksonomies has made this algorihm dominant in the Top- $\mathrm{N}$ tag based recomendations models.

While Vector Space model takes into account the individual annotation scores of users on items for computing personalized recommendations, in our opinion it only exploits a single dimension of these data.

Another interesting work which also employs cosine similarity is that of $\mathrm{Xu}$ et al. 32. They proposed a Tag-based CF system, which approaches the concept of tag-clustering, in which the cosine similarity of the frequency of each tag over the set of users is used to express a form of distance between the tags. In this way, a resource-tag matrix is composed out of the accumulated occurience rate of each tag, and is then used as input to a clustering algorithm. While the motivation of their approach is rational, finally allocating the similar tags into the same partition, however it does not take into account the semantic information carried by each tag. We mention this approach in our survey mainly because it follows a similar principle for similarity with that of the standard retrieval model for social tagging systems. 
Different from our concept, the work by Gemmell et.al [5], while it incoprorates the concept of clustering, it finally follows the well established principle used in the Vector Space model. In that one, item suggestions are made upon the computed relevance between users and items. Similarly to the work by $\mathrm{Xu}$ et al. 32] they perform clustering on the tags using as input their frequencies of usage in annotation excersizes.

In our opinion, it would not suffice if using alone the frequencies of tags in a metric of distance for tag clustering, because, frequencies can prove not enough to eliminate the implications of Synonimy and Polysemy. Polisemy exists because a tag might have multiple related meanings, and Synonimy exists when different tags sharing the same or similar meaning.

We should also mention the existence of works which explored the idea of studying the relationships between the tags in tag clouds. In the approach by Venetis et.al in 29. new metrics were introduced that capture those relationships. Nevertheless, in these works the concept of Annotation Competency is not approached towards a recommendation model.

\subsection{Semantic Similarity for Clustering}

The above points highlight the need to take into account the Semantic Similarity of tags in our model we introduce for personalized recommendations.

In the way it works, taxonomic similarity for tags works on static knowledge, which tags are as such by their nature. On the contrary, the computation of Cosine Similarity on the Vector Space model, being based on dynamic data, such as the item-tag data structure, requires recomputation upon the arrival of new data. The fact of taxonomic similarity of tags not being dependent on dynamic data, offers the practical advantage of such metric to work efficiently with complex algorithms, like Clustering. That means Clustering should need to run only once, since the distance between the tags does not change. Instead, in the Vector Space model, the frequency of each tag does change, as the RS system develops, requiring frequent recomputation of its value.

In a real system, it would suffice to run Clustering just once, during the system initialization, no matter how much data have been provided by the users for annotation. Furthermore, cold start issues would be avoided for the reason that the complete semantic network is established early on, during system initialization.

We believe that the concept of applying Clustering on the tag corpus, using the Semantic similarity as a metric for measuring the distance between the words used as tags, has truly strong potential. To the best of our knowledge, applying the above concept of Clustering for improving the performance of tag-oriented $R S$, while taking into account the users Annotation Competency, has not been investigated before. As far as the concept of Clustering is concerned, it has itself been the subject of research in RS for either improving the performance of predictions 22 22] or for securing RS against threats, such as profile injection attacks [14] 26] 34. 
In our opinion, to achieve a substantial benefit in terms of accuracy from the appplication of Semantic Clustering, it requires a model that would capture the characteristics of social tagging systems more sufficiently and that would incorporate the concept of Annotation Competency. As opposed to existing models, with our prediction model we attempt to utilize the potential of Collaborative Tagging by fusing our introduced properties of Diversity of Concepts and Annotation Contribution into the concept of Annotation Competency.

We sum up the main novelties introduced in our work to the following:

- A semantic-similarity based concept for partitioning the user tags.

- A model for computing personalized item recommendations that uses as input the Annotation Competency of users.

Considering the above requirements, in the next section we propose a model suitable for the tag-based $C F$ type.

\section{Proposed Model}

In this section, before we elaborate the details of our concept we will refer to useful knowledge about the components incorporated in our design. Moreover, we present the design considerations of our approach derived from the attributes and their requirements we set in the previous section.

\subsection{Concept Similarity}

In this section we describe in more detail the concept of Semantic Distance we will incorporate in our Similarity model. For computing the distance between any pair of tags we follow the intuitive idea of using the semantic similarity in a taxonomy. That is, the shorter the distance from one tag to another, the more similar the tags are. In our case, tags are regarded as nodes in the taxonomy tree. For computing the distance we used a metric introduced by Resnick [23, which is based on the notion of Information Content. According to this theory, the higher in the hierrarchy a concept is, the more abstract it is, and hence the less information it contains. Resnick's metric assumes the association of probabilities with concepts in a taxonomy and there is also an IS-A relatioship between them in the hierarchy. In that metric the similarity between two concepts $c_{1}$ and $c_{2}$ is given by:

$$
\operatorname{sim}\left(c_{1}, c_{2}\right)=\max _{c \in U\left(c_{1}, c_{2}\right)}[-\log p(c)]
$$

with function $p: C \rightarrow[0,1]$ such that for any concept $c \in C$ the value $p(c)$ to be the likelihood of encountering an instance of $c . p$ is monotonic such that, if $c_{1}$ IS-A $c_{2}$ then $p\left(c_{1}\right) \leq p\left(c_{2}\right) . U\left(c_{1}, c_{2}\right)$ denote as the set of concepts that subsume both $c_{1}$ and $c_{2}$ in the hierrarchy. The actual meaning of that equation is that the more infromation two concepts share in common, the more similar they are. 
The information shared by two concepts is indicated by the information content of the concepts that subsume them in the taxonomy.

To enchance clarity and provide a better understanding on how such model could adapt to the issue we come to address, we give an example. In the taxonomy of Fig, the similarity between felines and reptiles equals to similarities between tigers and snakes, as well as between tigers and reptiles $(\operatorname{sim}=5.19)$, for the reason that the set of concepts that subsumes both of them is $\{$ animals $\}$, and it is common for them. On the contrary, tigers are found to be more similar with cats $(\operatorname{sim}=10.15)$, than with bovines $(\operatorname{sim}=5.61)$. Tigers and cats are subsumed by felines, that is a concept of higher information content than mammals which subsumes tigers and bovines. Therefore, the similarity of the first pair has higher value than that of the second pair.

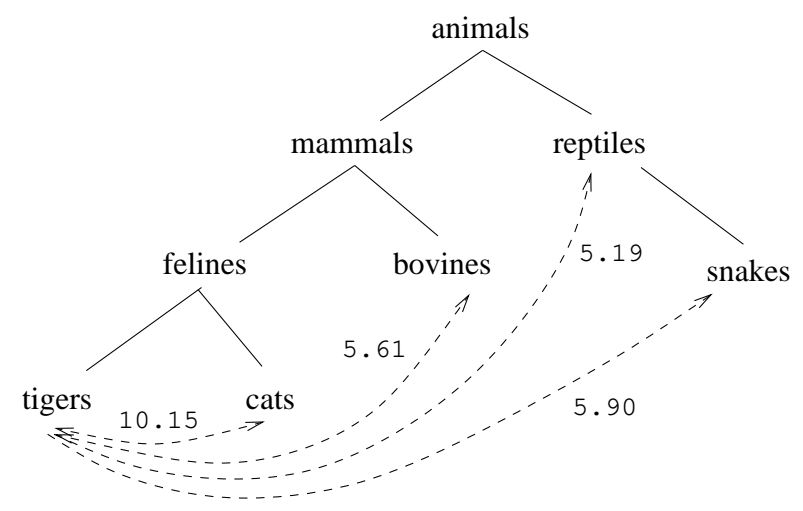

Fig. 2. Example taxonomy

\subsection{The Clustering Algorithm}

In order to partition the set of tags into clusters first and formost we needed a metric to express the distance between a pair of tags. We considered as distance the similariy values derived from the application of the Resnick similarity computed onto every pair of tags from a lexical database. WorldNet [3] is a large lexical database of the English language we chose for our experimentation. In WordNet the various parts of speech are grouped together into sets of cognitive synonyms, making up a network of meaningfully related words and concepts.

As far as the clustering algorithm to be used, we chose Affinity Propagation (AP), a newly developed clustering algorithm proposed by Frey et al. 4. AP was chosen as it is more efficient than other conventional approaches, such as $k$-means 12, and it has shown to achieve remarkably better clustering quality in various applications. For instance, when AP is applied onto a model for clustering the users of a collaborative filtering system, it helped to improve the prediction 
quality 22. AP is also known to achieve better performance than if using $K$ means for Abstracting data in anomaly Intrusion Detection Systems (IDS) [30].

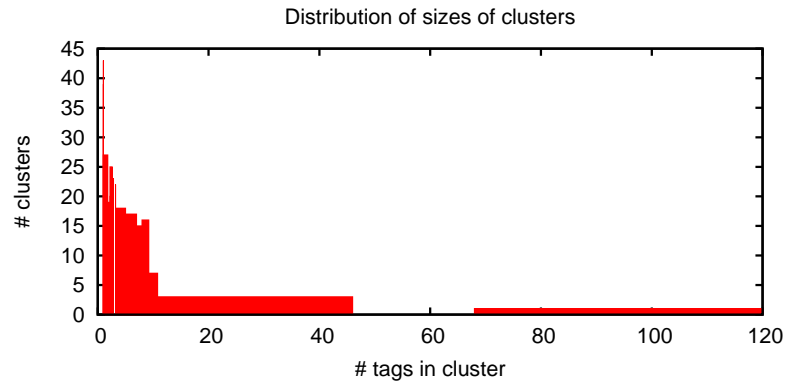

Fig. 3. Distribution of cluster sizes

Contrary to $k$-means algorithm, in which the number of clusters is predefined, in AP the quantity of clusters is dependent on the input data to be clustered, and it can also be affected by a value called Preference. That is a global value applied to each point expressing its suitability to serve as an exemplar. A big Preference value would cause AP to find many exemplars, while a small value would lead to a small number of clusters. Hence, the exact number of clusters emmerges deterministically after a few iterations of the algorithm. In our particular case, AP takes as input the similarities of the pairs of tags in the form of (tag1, tag2, similarity) which we considered as the data points to be clustered. For initial Preference value we chose the minimum similarity value, as that is the one suggested by the authors of the AP algorithm. For our dataset of 3162 tags, after the application of AP, 239 clusters were finally emmerged, 112 of which had been allocated one element only. We provide a graph of the distribution of the sizes of clusters in Fig 3 . Since the internals of tag clustering is out of the scope of our paper we will not describe the AP algorithm in more detail. The details of the AP can be referred to [4].

\subsection{System Model}

Assuming that all tags used by a user would portray his/her personal taste in annotation, it means that a proper analysis on the tags used by each user would reveal any hidden similarities that might exist between a user with others. Different from the existing approaches mentioned in the previous section, which mainly exploit the static knowledge provided by the users, in our proposed model 


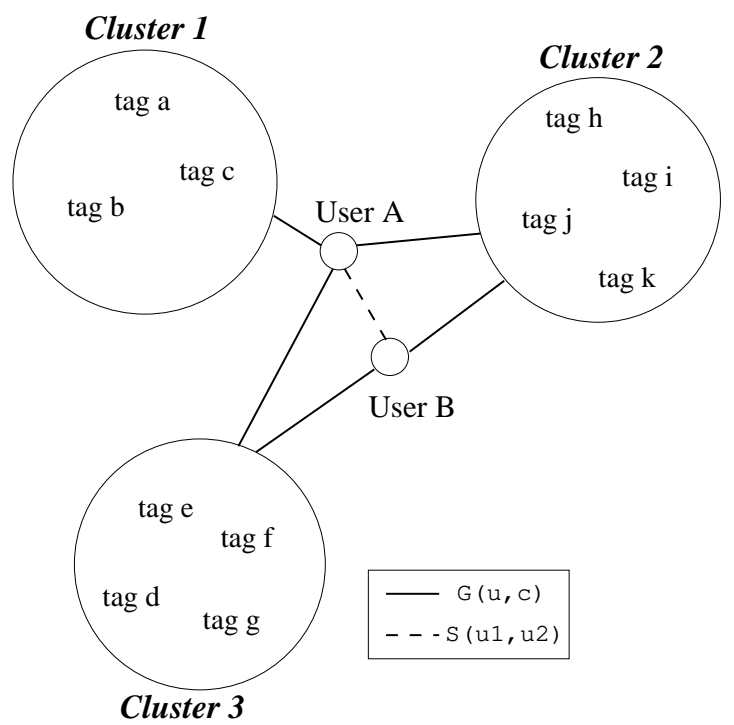

Fig. 4. Pictorial representation of our concept showing the entities involved.

instead there is an attempt to capture the characteristics of social tagging via the concept of Annotation Competency we set in section 2.2.

Examining the problem from a data perspective, the operations on those data can be divided into those applied onto the static ones, like the corpus of tags, and those applied onto the dynamic ones, which are related to the users' excercise on tagging.

In the high level view of our proposed idea that is shown pictorially in fig 4 can be distinguished, the available tags being clustered into separate groups, along with the distances between the users, denoted as similarity value $S(u 1, u 2)$. In the same figure, the relatedness of a user $u$ with some cluster $c$ is denoted as $G(u, c)$. Considering the above perception on data, Clusters represent the static, while the Sim and G refer to the dynamic part, which is derived from the user-item-tag association with the static data.

Next we define new metrics we use for capturing the central attributes of our concept of Annotation Competency. The metric of User Similarity shown in Def.1 captures the Diversity of Concepts expressed by the differences in the tastes of people and in their interests. The Diversity Value shown in Def.2 is another metric that captures the Diversity of Concepts on the user level. The metric of Tagging Effort in Def.3 captures the Annotation Contribution of users on particular items. With these 3 metrics combined together can be determined the suitable items to be recommended to a user.

Definition 1. To capture the central attribute of Diversity of Concepts in the tagging excercises on a pair of users $u_{i}$ and $u_{j}$ we introduce the metric of Similarity. 
Let's call $T$ the set of all tags used by all users. Tags are partitioned into $N$ clusters $c_{1}, c_{2}, \ldots, c_{N}$, with $C$ the set of all clusters $C=\left\{c_{1}, c_{2}, \ldots, c_{N}\right\}$.

We call $E\left(u_{i}, t_{j}, p_{k}\right)=\{0,1\}$ a function that specifies whether a user $u_{i}$ has tagged item $p_{k}$ using tag $t_{j}$.

We call $F$ a function that specifies whether a tag $t_{j}$ belongs to a cluster $c_{k}$ as:

$$
F\left(t_{j}, c_{k}\right)= \begin{cases}1 & t_{j} \in c_{k} \\ 0 & \text { otherwise }\end{cases}
$$

We introduce a function $G$, we call relatedness and it specifies whether a user $u_{i}$ is a member of cluster $c_{k}$ as:

$$
G\left(u_{i}, c_{k}\right)= \begin{cases}1 & \exists t_{j}, p_{k}: F\left(t_{j}, c_{k}\right)=1, E\left(u_{i}, t_{j}, p_{k}\right)=1 \\ 0 & \text { otherwise }\end{cases}
$$

The value of 1 is received only when the user in question has used at least one tag that belongs to that particular cluster in his annotation excersize.

Let the set of clusters by user $u_{i}$ be:

$$
C_{u_{i}}=\left\{c_{k} \mid c_{k} \in C: G\left(u_{i}, c_{k}\right)=1\right\}, C_{u_{i}} \subseteq C
$$

To express the similarity between two users $u_{i}$ and $u_{j}$ we adapted a function proposed by Jaccard [7] to the needs of our concept. The formula of user similarity is given in Eqn Jaccard metric is typically used in the field of data mining to measure the diversity or similarity in sample sets.

$$
S\left(u_{i}, u_{j}\right)=\frac{\left|C_{u_{i}} \cap C_{u_{j}}\right|}{\left|C_{u_{i}} \cup C_{u_{j}}\right|}
$$

The $\|$ indicates cardinality, (i.e., the number of clusters in the set). $S\left(u_{i}, u_{j}\right)$ has a range value in the interval $[0,1]$ and maximizes when the two sets $C_{u_{i}}$ and $C_{u_{j}}$ match. The intuition behind this formula is that the larger the number of common clusters the tags of the two users belong to, the more similar the users are, with regard to their taste in annotation and interests. For example, two users who are both interested in cars and machinery are expected to have used tags which belong to clusters most relevant to cars and machinery. Instead, if the first user has used tags that belong to a cluster that is more relevant to housing, it would be expected to have very low similarity with another user who has used tags which belong to both clusters of sports and leasure.

The central attribute of Diversity of Concepts within the tagging excersize of a single user is captured with the Diversity function we introduce next. The intuition behind this function is that, users whose interests comprise many subjects are meant to provide more valuable contribution, in comparison with other users, as far as the tagging excersize is concerned. Being classified as the most important ones, the opinions of those users will be taken into account for rec- 
ommending articles to others.

Definition 2. We propose the following function for Diversity Value $w\left(u_{i}\right)$, which returns a binary quantity, by which we classify whether a user's tagging contribution is valuable or not.

We define $U_{h} \subset U, U_{h}=\left\{u_{1}, u_{2}, \ldots, u_{k}\right\}: k \in[1, . .,|U|]$, an ordered subset of all users set $U$, such that for any two users $u_{f}, u_{g} \in U_{h}$ and $\forall f, g \in[1, \ldots, k]$, with $k=|U|$, for which $\left|C_{u_{f}}\right|<\left|C_{u_{g}}\right| \Rightarrow f<g$. We call $U_{h_{1}}$ an ordered subset of $U_{h}$, so that $U_{h_{1}}=\left\{u_{1}, \ldots, u_{h}\right\}$ with $h=\frac{|U|}{2}$. The Diversity function returns the value:

$$
w\left(u_{i}\right)= \begin{cases}1, & u_{i} \in U_{h_{1}} \\ 0 & \text { otherwise }\end{cases}
$$

The binary value received from Diversity function $w\left(u_{i}\right)$ is finally used for filtering out the poorly experienced users, judged on objective criteria. More particularly, a user that belongs to the top $50 \%$ of the most experienced ones, in terms of diversity in the subjects of interest, would be considered as a highly contributing user. In our concept, every distinct area of interest is assumed to belonging to a different cluster.

As we mentioned, the above two metrics of Diversity in Def.2 and Similarity in Def.1, are computed upon both the static and dynamic portion of data, and therefore their values require recomputation as the user experiences grow.

In order to be in line with the second property of our design, which we called Annotation Contribution, we adopt the following intuition: We consider those users who have put more effort in annotating some particular items, as being the strongest candidates for recommeding these items to other users. To be consistent with our desing principals, the prediction mechanism should be more sensitive to the quantity and the diversity of tags used by some user for annotating a particular item. Therefore, we find nessasary to introduce the notion of Tagging Effort, that we express here in the form of a metric and we use it as a complementary criterion for filtering out the items of lower interest from being recommended to users.

Definition 3. We define the metric of Tagging Effort, $f$ on some item $p_{k}$ as follows. We call $T$ the set of all tags used by all users and $T_{u_{i}} \in T$ the set of tags used by a particular user in his annotation excersize. We call $T_{\left(u_{i}, p_{k}\right)} \in T_{u_{i}}$ the subset of tags used by $u_{i}$ for tagging item $p_{k}$. Tagging Effort $f\left(u_{i}, p_{k}\right)=\frac{\left|T_{\left(u_{i}, p_{k}\right)}\right|}{\left|T_{u_{i}}\right|}$ is defined as the fraction of tags used by user $u_{i}$ for tagging the candidate item $p_{k}$ over all tags used by that user.

To investigate the level of contribution for each of the two criteria of Diversity of Concepts and Annotation Contribution into the quality of predictions, we introduce the contribution factor $d$. We use the following formula to combine together the above two criteria expressed as per metrics of User Similarity and 
Tagging Effort. The probability of an item $p_{k}$ to be recommended to user $u_{i}$ by another user $u_{j}$ is computed as:

$$
p\left(u_{i}, u_{j}, p_{x}\right)=d \cdot S\left(u_{i}, u_{j}\right)+(1-d) \cdot f\left(u_{j}, p_{k}\right)
$$

Finally, the probability of an item $p_{k}$ to be liked by user $u_{i}$ is given in equation 7 and it represents the normalized liking of the particular item over all $m$ users who have also experienced the item $p_{k}$.

$$
p\left(u_{i}, p_{x}\right)=\frac{\sum_{j=1}^{m}\left[p\left(u_{i}, u_{j}, p_{x}\right) \cdot w\left(u_{j}\right)\right]}{m}
$$

\section{Evaluation}

For computing the similarity between the tags of users we used Wordnet::Similarity, a freely available software package by Pedersen et al. [17, that is written in perl. This package provides various measures of relatedness including Resnick's metric which we finally chose to use in our experiment. WordNet::Similarity implements the similarity proposal for IS-A relationships in 23].

We chose the CiteUlike dataset as the most appropriate set for our evaluation. CiteUlike is a public social bookmarking site aiming to promote and develop the sharing of scientific references amongst researchers. One can add a scientific references and then add tags of his choice, allowing to other users to search for references by keywords. The data we used was taken from an available snapshot retrieved in 2009 from the CiteUlike website, and that is provided for research purposes [1. This dataset is available in the form of a single file, every line of which is consisted of four elements: a) the id of an article annotated, $b$ ) the ID of the user who annotated the article and $c$ ) the tag word used for annotation, and $d$ ) the time of the annotation. From the above fields we can easily build the associations between users, tags and articles. For the needs of our experiment, and due to the fact that the original dataset was very large and sparse, we finally chose a subset of 1000 users, randomly selected out of the 46444 users contained in the original set.

For computational efficiency and recommendation quality we applied filtering onto the selected articles so that only those which have been annotated by at least 15 users were finaly considered in the evaluation. In addition, articles that had been annotated for more than 75 times were excluded. For the same reason we applied filtering on the tags too, considering only those that have been used for at least 10 times in the training set. Finally, we also applied filtering on the users set. Thus, the final 1000 user dataset we used, contained only users who had annotated at least 20 articles. We chose these filtering values in order to minimize the impact of the use of reduced dataset on the tested algorithms.

We performed 5-fold cross validation over the 1000 user data set to test all algorithms. That is, we randomly divided the user dataset into 5 subsets of 200 users each, where in each fold we kept the annotations of one subset of users 
hidden and tried to predict the liking of those users, using the remaining 4 folds. The former and the latter subsets are known as test set and training set respectivelly. In the prediction phase we used our algorithm to recommend the top 20 articles for each user, and compare them with the actual articles found in the test set for the same user.

To distinguish the probable articles we set a Threshold Probability value of zero as the probability of an article must not be equal to, in order to be counted as a probable article overall. Then, for every user we compiled a top 20 list which includes those articles whose predicted probabilities to be liked by that user would have exceeded the Threshold Probability value. We call a hit an article which has been selected in the top 20 list of a user and for which it trully happens to be one of the items which the user has annotated. We assume that users only annotate items which they trully like. We measure the number of correct recommendations, or hits with the symbol $N_{\text {hit }} . N_{\text {rec }}$ is the total number of recommendations and it counts those cases in which the computed probability $p\left(u_{i}, p_{x}\right)$ in eq. 7 has received a positive value. $N_{\text {test }}$ is the number of articles in the test set. After applying the filtering there were in total 845 articles found to meet the criteria, which composed our test set $\left(N_{\text {test }}=845\right)$. More details about the data used can be found in table 1 .

Table 1. Data description table. *A transaction indicates an instance of a single tag out of all tags used by some user for annotating an article.

\begin{tabular}{|c|c|}
\hline Metric & Value \\
\hline \hline Number of users after applying filtering & 518 \\
\hline Number of articles after applying filtering & 845 \\
\hline Number of total tags used by filtered users & 3162 \\
\hline Number of transactions & 4931 \\
\hline Avg. Number of articles per user & 1.631 \\
\hline Avgerage frequency of selected tags & 3.062 \\
\hline Number of clusters of Tags & 239 \\
\hline
\end{tabular}

Moreover, we performed further analysis onto the results for the proposed algorithm, measuring the performance for the highly active and the least active users in separate. For classifying the highly active users we used the Median of the number of clusters $\left|C_{u_{i}}\right|$ of each individual user as a threshold value. As defined earlier in Def.1, $C_{u_{i}}$ is the the number of clusters which the tags of user $u_{i}$ are spanning to, meaning that a user with $\left|C_{u_{i}}\right|$ value larger than the chosen threshold would be considered as a highly contributing user and hence as a highly active one.

For measuring the ability of our proposed algorithm to provide a list of recommendations of articles that users actually like, we used the evaluation method called Precision and Recall. This method measures this ability in terms of Classification Accuracy and it is widely used in Information Retrieval [6] 13. The metrics used in Classification accuracy are Precision (P), Recall (R) and 
$F \_S c o r e(\mathrm{~F})$. For the case of systems that generate $T o p_{-} N$ recommedations, like ours, the definitions of Precision and Recall are slightly adjusted from the standard way used in Information Retrieval.

Precision indicates the success of the algorithm regarding whether some recommendation provided by the algorithm for some particular user matches a real liking of that user. Precision is defined as the ratio of $\frac{\text { size of hit set }}{\text { size of top_N set }}$. The relative success in retrieving all items liked by individual users is expressed with Recall. Finally, the trade-off between $\mathrm{P}$ and $\mathrm{R}$ is measured with the F_score, which is the harmonic mean of the two values. The metrics used are shown in table 2.

Table 2. Evaluation Metrics

\begin{tabular}{|c|c|}
\hline Metric & Formula used \\
\hline Precision & $\frac{\text { size of hit set }}{\text { size of top_N } \text { set }}=\frac{N_{\text {hit }}}{N_{r e c}}$ \\
\hline Recall & $\frac{\text { size of hit set }}{\text { size of test set }}=\frac{N_{\text {hit }}}{N_{\text {test }}}$ \\
\hline F.score & $\frac{2 \cdot \text { Precision } \cdot \text { Recall }}{\text { Precision }+ \text { Recall }}$ \\
\hline
\end{tabular}

For reference we also tested the classical Vector Space method onto the same data, which employs no clustering. To perform this we computed the cosine similarity as in eqn 3 between every pair of users and items and we finally selected a list of Top_20 most similar items to recommend for each user. We also compared against the while simple, but powerful alternative Tag-Based recommendation method by Peng et al., expressed in eqn. 1, For comparison and for showing whether the use of the transition probability over all subjects might work better with clustering, we also evaluated the Topic-Based variation by Peng et al., expressed in eqn 2. Finally, in our evaluation we used our clustering approach for deriving the various subject categories.

To reason whether prediction schemes do actually worth over doing selections without definite aim, we also tested our method against a random selection scheme. The main idea of random selection scheme is to build up the lists of recommended items for each user, in which the top items (20 in our experiment) will be randomly selected out of the $N_{\text {test }}$ items of the whole set. Next, the number of correct recommendations $N_{\text {hit }}$ is counted for each user as normal.

\section{Results - Discussion}

We report the most interesting results of our experimentation. The data presented in table 3 shows the average values of 10 measurements. The largest values of $P, R$ and $F_{\text {_score }}$ are highlighted in bold. We tested our scheme for various values of the $d$ factor, ranging from 0.0 to 1.0 . This was mainly done to study whether a mixture of the two criteria has any effect on the prediction quality. We also include in our report the performance figures of Tag-based Recommendation method by Peng et.al [19], and its variation we called Topic-based 
Recommendation [18, that we compare ours against to. All comparative results are shown pictorially in fig 516 and 7

Table 3. Numeric Results

\begin{tabular}{|c|c|c|c|c|c|}
\hline Method & $\mathrm{R}$ & $\mathrm{P}$ & $\mathrm{F}$ & $\mathrm{F}$ (most active) & $\mathrm{F}$ (least active) \\
\hline \hline Random Choice & 0.001593 & 0.04874 & 0.003084 & - & - \\
\hline Peng et al. & 0.001636 & 0.10046 & 0.003190 & - & - \\
\hline Peng et al. (topic-based) & 0.001797 & 0.05881 & 0.003485 & - & - \\
\hline Vector Space. & 0.001883 & $\mathbf{0 . 2 4 2 4 2}$ & 0.003685 & - & - \\
\hline proposed, $d=0.0$ & 0.001874 & 0.09176 & 0.003641 & 0.003967 & 0.003325 \\
\hline proposed, $d=0.1$ & 0.001851 & 0.08408 & 0.003594 & 0.003939 & 0.003262 \\
\hline proposed, $d=0.2$ & 0.001892 & 0.09261 & 0.003678 & 0.003976 & 0.003395 \\
\hline proposed, $d=0.3$ & 0.001743 & 0.09295 & 0.003393 & 0.003733 & 0.003070 \\
\hline proposed, $d=0.4$ & 0.001748 & 0.10385 & 0.003404 & 0.003728 & 0.003102 \\
\hline proposed, $d=0.5$ & 0.001798 & 0.11824 & 0.003404 & 0.003965 & 0.003086 \\
\hline proposed, $d=0.6$ & 0.001815 & 0.11714 & 0.003539 & 0.003829 & 0.003275 \\
\hline proposed, $d=0.7$ & 0.001878 & 0.12024 & 0.003658 & 0.003919 & 0.003412 \\
\hline proposed, $d=0.8$ & 0.001987 & 0.12815 & 0.003859 & 0.004099 & 0.003650 \\
\hline proposed, $d=0.9$ & $\mathbf{0 . 0 0 2 2 2 1}$ & 0.09837 & $\mathbf{0 . 0 0 4 3 1 8}$ & $\mathbf{0 . 0 0 4 5 7 7}$ & $\mathbf{0 . 0 0 4 0 7 6}$ \\
\hline proposed, $d=1.0$ & 0.002019 & 0.12618 & 0.003925 & 0.003775 & 0.004070 \\
\hline
\end{tabular}

We make the following observations on the results. As can be seen in figures 516 and 7, our method outperforms all the alternative algorithms we compared against it. More specifically, our method performs best when the $d$ factor receives extreme values $(b \rightarrow 0$ or $b \rightarrow 1$ ). Moreover, the proposed method outperforms all the other alternatives in terms of $F_{-}$Score, when $d>0.7$, with performance reaching its peek for $d=0.9$. According to our results, the Vector Space model is the second best performing alternative, with the third best to be the Topicbased recommendation method by Peng et.al., which our method outperforms for almost all values of $d$, (except for $d=0.3$ and $d=0.4$ ). The observed peak value for $F_{-}$score at $d=0.9$ can be interpreted as saying: in our proposed method the Tagging Effort criterion on particular items is less significant than that of Profile Similarity.

More precisely, at that peak value of $F_{-} S c o r e$, our method $(d=0.9)$ appears to be $17.17 \%$ better than the second best (Vector Space model) achieving $F_{\_}$score $=0.00432$ vs 0.00368 .

From the diagrams of $P$ and $R$ in fig 5 and 6 , we observe that the good performance of our method is terms of $F$, in relation to the other methods we compared it with, is due to the high $R$ values achieved. If considered the Precision values alone, the Vector Space model would have been the best performed. On the contrary, in terms of Precision, the value of $d$ does not appear to have the same strong impact on the performance as it does for Recall.

Moreover, looking at the diagrams of Precision and Recall more carefully, we can observe a significant drop in the Precision values for a long range of 


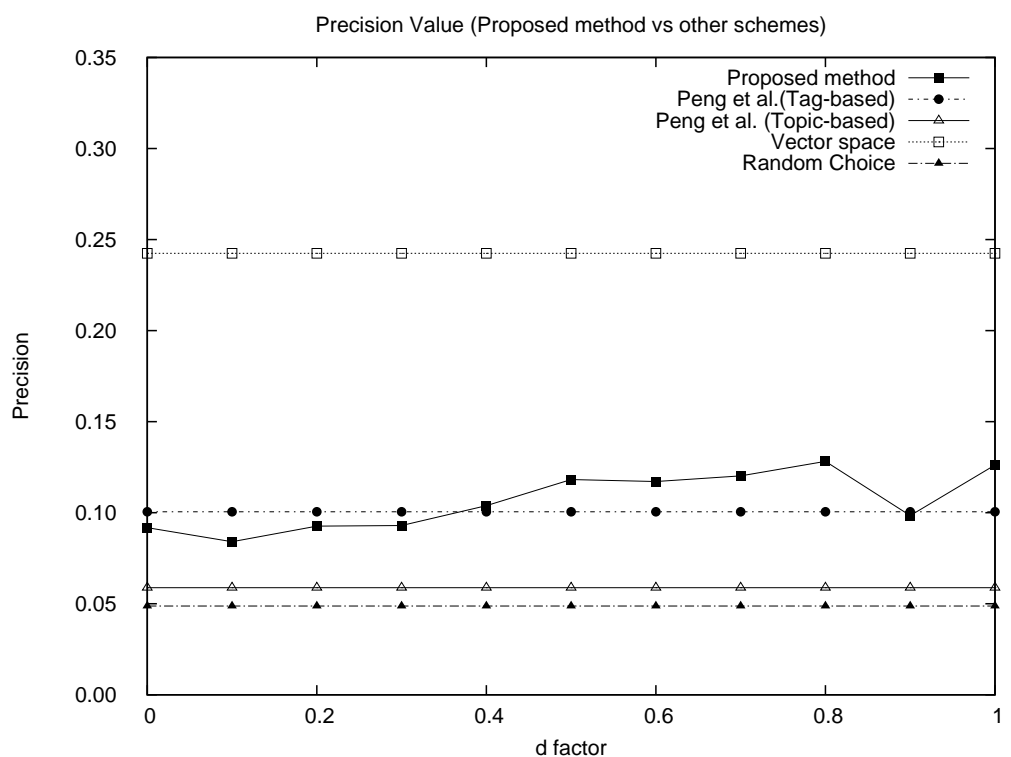

Fig. 5. Comparison of the proposed method against other algorithms in terms of Precision

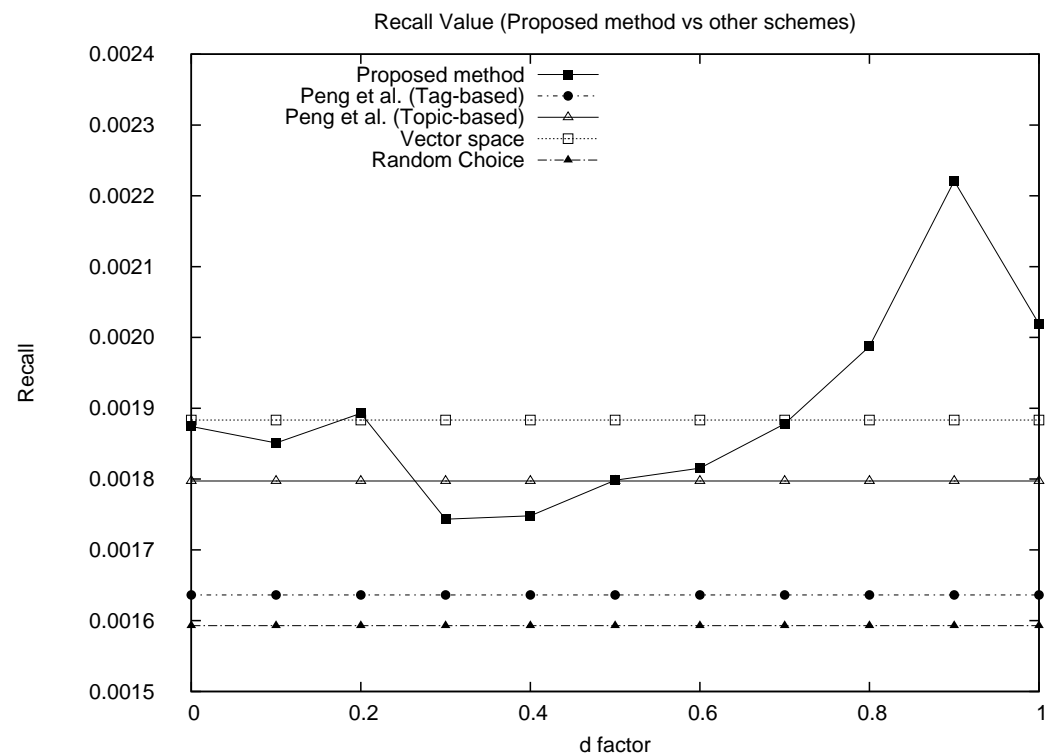

Fig. 6. Comparison of the proposed method against other algorithms in terms of Recall 


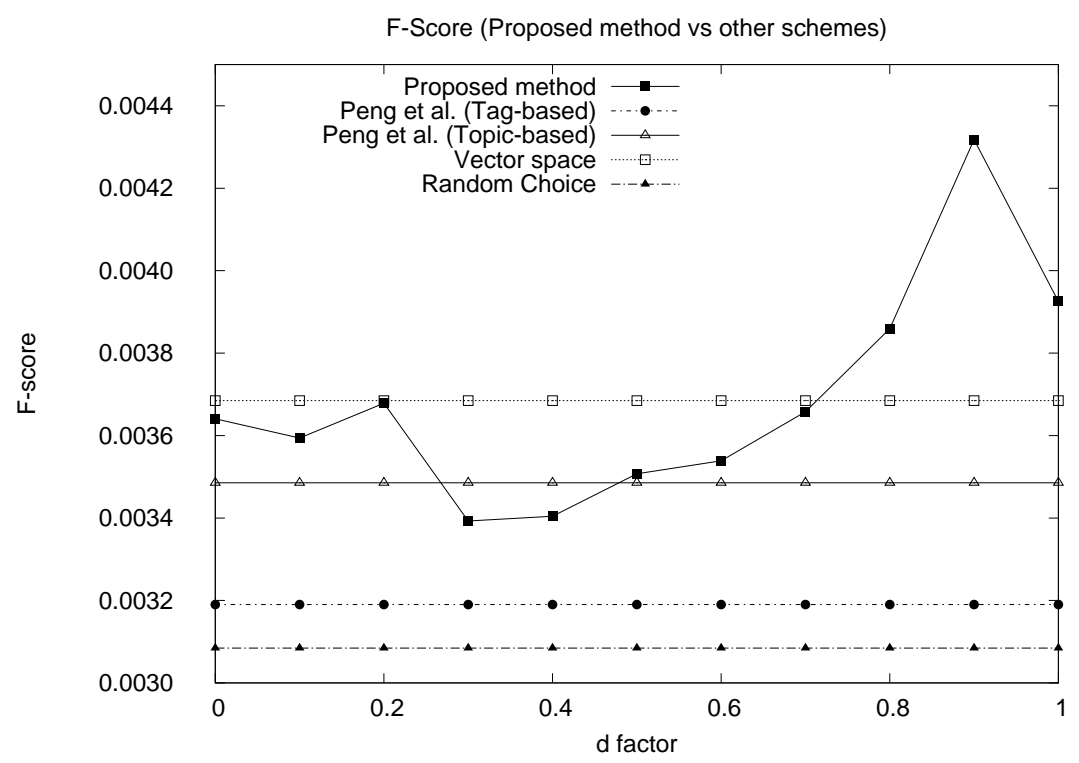

Fig. 7. Comparison of the proposed method against other algorithms in terms of F_score

the $d$ factor. Nevertheless, this drop seems to be not enough to eradicate the advantage of Recall values in our method, which achieved for large values of $d$. This observation can be interpreded as saying that the Profile Similarity criteria is more important for achieving good predictions. On the contrary, in terms of Precision, the value of $d$ does not have a strong impact on the performance.

Comparing against the method by Peng et.al alone, we conlcude that,in overall, their both variations produced significantly lower figures of performance than ours, in all aspects.

To investigate the distribution of the $F_{-}$Score in the number of users, we also demonstrate the Cumulative Distribution Function (CDF) of F_Score in figure 8. CDF describes the probability that $F_{-} S$ core receives a value less or equal to $\mathrm{x}$ $\left(\operatorname{Pr}\left(F_{-}\right.\right.$score $\left.\left.\leq x\right)\right)$. In a good model, $F \_s c o r e$ would receive as large as possible values, meaning that the CDF curve should go up as less quickly as possible for a good model, meaning that a curve close to the right-bottom corner of the diagram indicates a good model. In general, a CDF curve that is away from the left-top corner of the diagram indicates a good model. In figure 8 we present the CDF of the F_Score of our proposed method, as well as the two variations of the Peng et al. technique, and the Vector Space model. We also include the performnce of the Random selection scheme for recommending items.

In total we test our proposed technique for 3 different values of the $d$ factor, 0.0, 0.9 and 1.0. The choice of values for the $d$ factor was done using the following reasoning: 1.0 and 0.0 were chosen as the extreme values which indicate the sole application of either of the two criteria of Tagging Effort or Profile Similarity 
The value 0.9 was chosen as an intermediate case in which the method behaves best in terms of Classification Accuracy. We observe that our proposed method performed best in terms of CDF, only for the case that a mixture of criteria was applied. More particularly, the best results achieved for $d=0.9$.

Compared to the alternative methods we included in our evaluation, we observe that our proposed technique has shown the best behaviour, with Vector Space and Topic-Based model by Peng et. al to have achieved poor results. Given a scenario that $F_{-} S c o r e$ would not fall below than 0.0075 , in our proposed technique only the $99.27 \%$ (for $d=0.9$ ) of the users would behave as such. That is to say: the F_Score has $99.27 \%$ chance to not exceed the value of 0.0075 , while for the vector space model, the chance of exceeding the same value is even larger (99.55\%). In the method by Peng et al, as well as in their Topic-Based variation that makes use of subjects, the chance is also higher, (99.9\% for both). For the random policy model, that chance reaches the $100.0 \%$. Compared to each other in therms of CDF, our technique in overall (Considering all cases where F_score $<x)$ produces better results, by just $0.126 \%$ than the vector space model, $0.412 \%$ than the peng et al. method, and $0.389 \%$ than the subjects version of the algorithm by the same authors.

Despite the marginal superiority of our method, we can intrepret the promishing results of our approach as saying: In our method it is more likely for the F_Score to receive higher value than in any other model used in our experiment.

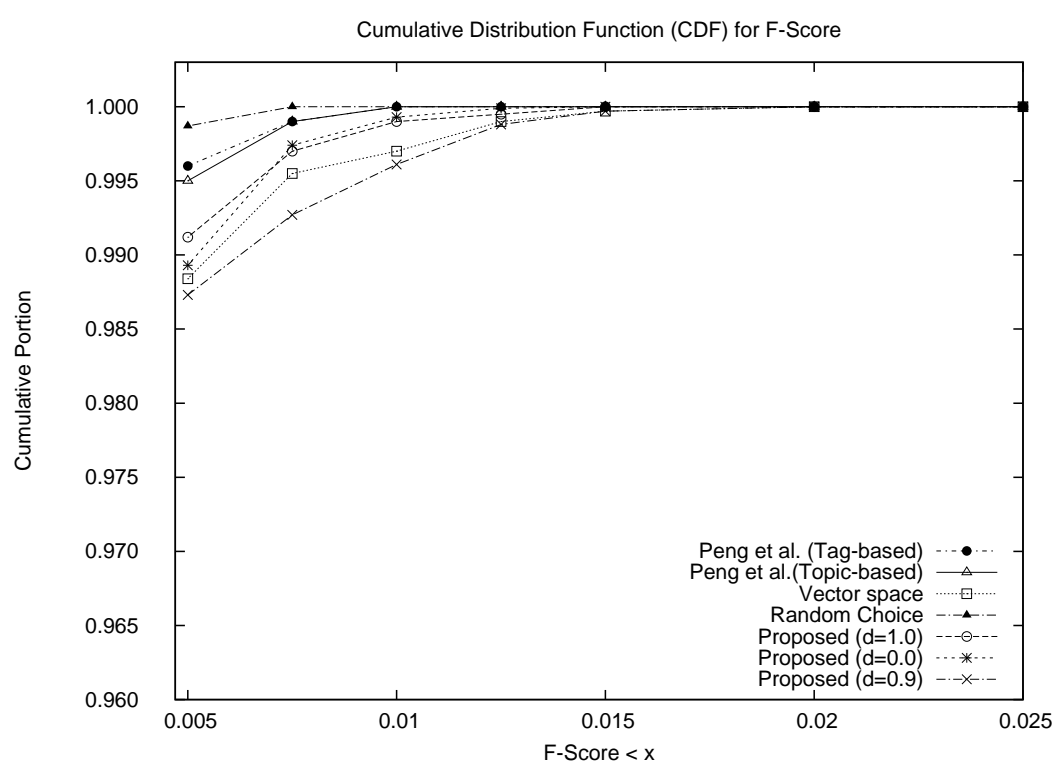

Fig. 8. CDF of F.Score in the proposed method against other algorithms 
We also present separate performance figures in table 3 and fig. 9 for specific classes of users, like the Most active and the Least active ones.

For the class of Most Active users our method does significalty better than for the mixed population and it outperforms all the approaches it was tested against in terms of $F_{-} S$ core in the whole range of $d$ values. As can be seen the best performance is achieved when a mixture of criteria is applied $(d=0.9)$, achieving $19.50 \%$ better accuracy than the second best approach, (Vector Space model). It is interesting to note that this value is the highest ever counted for all categories of users we tested (Most active, Least active and the mixed group of users). On the contrary, for the Least actrive users, the performance of our method produced a lower figure, but it still outperformed the Vector Space for $d>0.8$. More specifically $F \_s c o r e$ ranged from performance levels as low as that of the random choice $(d=[0.3, \ldots, 0.5], \mathrm{F}=0.003084)$, but finally achieving the best performance $(\mathrm{F}=0.004070)$ for that category of users for $d=1.0$.

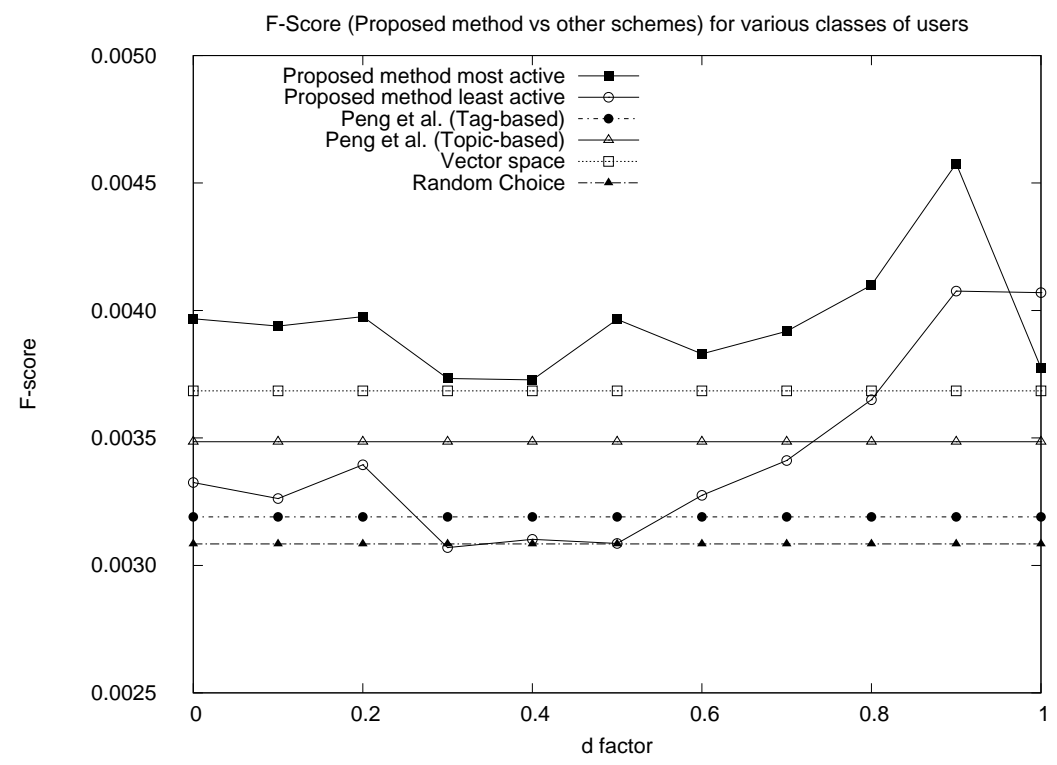

Fig. 9. Comparison of the proposed method against other algorithms for various classes of users

Beside Classification Accuracy, it is equally important to know the success of the method as far as the population of users that can actually receive the recommendation service. With Covered Population we refer to the users who were able to receive at least one recommendation for their articles included in the test set. Data sparsity is the reason that not all 1000 users from the sample could finally receive recommendations. We present the results of Covered Population for all algorithms we compared against in table 4 and in fig 10, As 
can be seen the Covered Population is indeed affected by the use of clusters. On the contrary, the Topic-based variation of the algorithm by Peng et al. is affected the most, allowing only to the $5.6 \%$ of the total population (56 over 1000 users) to receive recommendations. Instead the Vector Space method is the least affected in terms of coverage. As we can observe, our method is becoming more sensitive with the increase of $d$, achieving Coverage Population that ranges from $103.9(d=0)$ to $56.6(d=1)$. At the peak performance in terms of $F_{-} S c o r e$ $(d=0.9)$, the Coverage of our method shows a significant drop, as opposed to the Vector Space model, with the number of users who can accually receive recommendations to have been reduced in half (62.3 vs 120.7).

Table 4. Coverage over 1000 users population

\begin{tabular}{|c|c|}
\hline Method & $\begin{array}{c}\text { Covered } \\
\text { Population }\end{array}$ \\
\hline \hline Random Choice & 47.0 \\
\hline Peng el al. & 106.9 \\
\hline Peng et al. (Topic-based) & 56.0 \\
\hline Vector Space & 120.7 \\
\hline proposed, $d=0.0$ & 103.9 \\
\hline proposed, $d=0.1$ & 103.7 \\
\hline proposed, $d=0.2$ & 93.0 \\
\hline proposed, $d=0.3$ & 91.8 \\
\hline proposed, $d=0.4$ & 92.0 \\
\hline proposed, $d=0.5$ & 85.2 \\
\hline proposed, $d=0.6$ & 75.9 \\
\hline proposed, $d=0.7$ & 74.0 \\
\hline proposed, $d=0.8$ & 69.4 \\
\hline proposed, $d=0.9$ & 62.3 \\
\hline proposed, $d=1.0$ & 56.6 \\
\hline
\end{tabular}

In addition, the figures show that the clustering algorithm we chose (Affinity Propagation) applied partitioning onto the tags in a way that resulted to receiving a large number of small clusters, making the computation of Similarity betweed users less probable. That posed a serious implication on the number of the computed recommendations. As such, the chance for the topics of users' interests to overlap is reduced. For that reason we conclude that clustering with Affinity Propagation is not suitable for the Topic-based variation method by Peng et al. (see eqn 2), while for our method it worked beneficially for a whole range of values of $d$. For example, for $d \geq 0.5$ our method does better both in terms of Coverage and F_Score, against the Topic-based by Peng et. al. The higher performance achieved by our technique, which also employs partitioning for distinguishing the subjects used, justifies the importance of the notions of Diversity of Concepts and Annotation Contribution we introduced. 


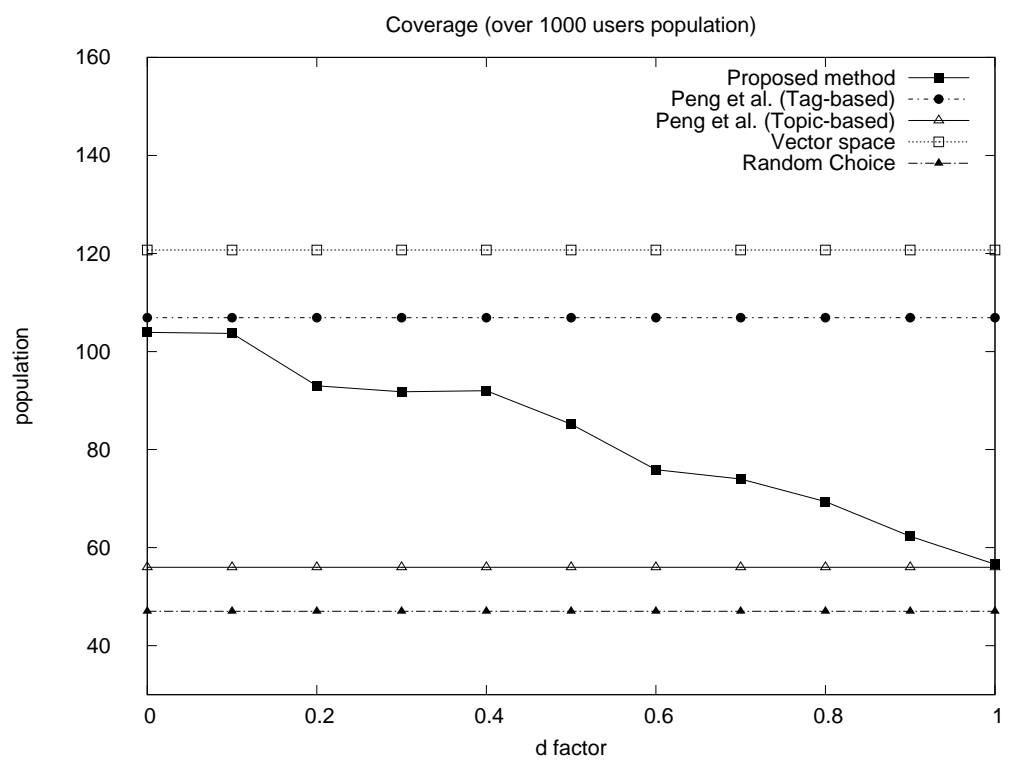

Fig. 10. Comparison of the proposed method against other algorithms in terms of Coverage

The computational cost of our approach to generate recommendations for a user comes from the participation of the following 3 factors:

- User Similarity computation - (as per eqn 5 )

- Tagging Effort of a user to particular item - given by $f\left(u_{i}, p_{k}\right)$ (as per Def.3)

- Probability for an item to be liked by a user - (as per eqn 7 ).

We used the following notation, with $m$ denoting the users, $n$ the items, $l$ the tags and $c$ the clusters. As such, the time complexity of the similarity computation is $O(c(m+m))$. Similarly, the time complexity of the Tagging effort is $O(l+n l)$ and the complexity of computing the probability of liking for an item is $O(m)$. In overall, the complexity of our model is $O(m[2 c(m+m)+l+n l])=$ $O(m(\mathrm{~cm}+n l))=O\left(m^{2} c+m n l\right)$. Respectivelly, for the conventional method (Tagbased ) by Peng et al. expressed in eqn 1 the complexity is $O(l(n+n l+m+m l))$ $=O\left(l^{2} n+l^{2} m+l n+l m\right)$. As can be seen, the second parts in the two expressions (ours and Tag-Based Pend et al.) denote complexity of equal degree (3rd). The complexity of Topic-based variation of Peng et al. method is $O(c(n+n c+m+m c))$ $=O\left(c^{n}+c^{2} m+c n+c m\right)$, and it is nearly equal to that of the first variation, if not including the cost of clustering.

Likewise, the complexity of the vector space method is computed as follows: The time compexity of the task of computing the tag frequency tables over all users and all articles are: $O(l m)$ and $O(\ln )$ respectivelly. The cosine similarity 
computation itself adds another $O(3 \mathrm{lmn})$ time complexity to the method, when applied onto all pairs of users and articles, while adding up another $O(\mathrm{~nm})$ for the construction of the top lists. In overall for the Vector Space model the complexity is $O(l m+\ln +3 \ln m+n m)=O(\operatorname{lnm})$. As can be seen, our method again does not exceed the complexity levels of the classical vector space method.

In our opinion, the large overhead generated by the tag clustering process is not that serious for causing any applicability issues in our method. Such overhead is mainly caused by the fact that the input data used for expessing the distances between the tags are not derived directly from the user's tagging experience, as it is the case for other traditional methods, such as Vector Space, or the method by Peng et al. In our method instead, clustering is computed upon the semantic similarity of tags, and for that reason clustering data remains constant thereafter. Therefore, such cost does not contribute to the computational complexity of the recommendation process. For that reason it sufficies if applying pre-clustering once, upon system initialization, and then using the clustering data to any predictions computed thereafter. On the contrary, any approaches based on Vector Space model would require re-computation of clusters on a regular basis, as the user data change, resulting to significant overhead in the system.

\section{Conclusions and Future work}

Annotation Competency of users has very little been explored in Recommender Systems. In this paper we attempted to explore the potential of using the information derived from the Annotation Competency of users for improving the prediction accuracy of a Tag-based Recommender system. Such type of systems use alone the tags provided by users for computing personalized item recommendations. Prior works on tag-based recommendations have indicated that there was still space for improvement. Our work is motivated by the need to better understand how users' annotation works and it provides a new insight on how such knowledge could be incorprorated into the mecanism of producing personalized recommendations. We introduced a new approach which applies clustering onto the set of tags that works in succession with our proposed formula for predicting recommendations. Our formula takes into account the properties of Diversity of Concepts and Annotation Contribution we introduced for describing the notion of Annotation Competency. We attempted evaluation on our proposed model using data from citeUlike, a public annotation system for scientific documents. Our experimentation showed that, if the above two properties are put together, it can help substantially to increase the benefit expressed in terms of recommenations quality for users. At the same time, the proposed method was found to be equally computational efficient with other baseline approaches.

We believe that our work will make significant impact on on-line Searching and Recommendation services as its simplicity and its low overhead makes it suitable for such services. We note the importance of getting a better undestanding of the mechanism of the users' annotation excersize. A wider comparison against 
more Tag-based recommendation algorithms is left as future work. Another important future work is to confirm our conclusions on more annotation datasets. Investigating our method from the security point of view is also an interesting research direction.

\section{References}

1. Citeulike: A researcher's social bookmarking service. Ariadne: Issue 51.

2. T. DuBois, J. Golbeck, J. Kleint, and A. Srinivasan. Improving Recommendation Accuracy by Clustering Social Networks with Trust. In ACM RecSys'09 Workshop on Recommender Systems $\&$ the Social Web, Oct. 2009.

3. C. Fellbaum. Wordnet and wordnets. In K. Brown, editor, Encyclopedia of Language and Linguistics, pages 665-670, Oxford, 2005. Elsevier.

4. B. J. Frey and D. Dueck. Clustering by passing messages between data points. Science, 315:972-976, 2007.

5. J. Gemmell, A. Shepitsen, M. Mobasher, and R. Burke. Personalization in folksonomies based on tag clustering. In Proceedings of the 6th Workshop on Intelligent Techniques for Web Personalization and Recommender Systems, July 2008.

6. J. L. Herlocker, J. A. Konstan, L. G. Terveen, John, and T. Riedl. Evaluating collaborative filtering recommender systems. ACM Transactions on Information Systems, 22:5-53, 2004.

7. P. Jaccard. Distribution de la flore alpine dans le bassin des Dranses et dans quelques régions voisines. Bulletin de la Société Vaudoise des Sciences Naturelles, 37:241-272, 1901.

8. R. Jaschke, R. Marinho, A. Hotho, L. Schmidt-thieme, and G. Stumme. Tag recommendations in folksonomies. In In PKDD, pages 506-514. Springer, 2007.

9. J. Li and J. Z. Wang. Real-time computerized annotation of pictures. IEEE Trans. Pattern Anal. Mach. Intell., 30(6):985-1002, June 2008.

10. Y. Li, H. Liang, R. Nayak, and Y. Xu. Collaborative filtering recommender systems using tag information. In Y. Li, G. Pasi, C. Zhang, N. Cercone, and L. Cao, editors, Proceedings of the 2008 IEEE/WIC/ACM International Conference on Web Intelligence, pages 59-62, Australia, New South Wales, Sydney, 2008. The Institute of Electrical and Electronics Engineers, Inc.

11. M. Lipczak and E. Milios. Learning in efficient tag recommendation. In Proceedings of the fourth ACM conference on Recommender systems, RecSys '10, pages 167174, New York, NY, USA, 2010. ACM.

12. J. B. MacQueen. Some methods for classification and analysis of multivariate observations. In L. M. L. Cam and J. Neyman, editors, Proc. of the fifth Berkeley Symposium on Mathematical Statistics and Probability, volume 1, pages 281-297. University of California Press, 1967.

13. S. M. McNee, J. Riedl, and J. A. Konstan. Being accurate is not enough: how accuracy metrics have hurt recommender systems. In CHI 'O6 extended abstracts on Human factors in computing systems, CHI EA '06, pages 1097-1101, New York, NY, USA, 2006. ACM.

14. B. Mehta. Unsupervised shilling detection for collaborative filtering. In Proceedings of the 22nd national conference on Artificial intelligence - Volume 2, AAAI'07, pages 1402-1407. AAAI Press, 2007.

15. E. Moxley, J. Kleban, and B. S. Manjunath. Spirittagger: a geo-aware tag suggestion tool mined from flickr. In Proceedings of the 1st ACM international conference 
on Multimedia information retrieval, MIR '08, pages 24-30, New York, NY, USA, 2008. ACM

16. D. Parra and P. Brusilovsky. Collaborative filtering for social tagging systems: an experiment with citeulike. In L. D. Bergman, A. Tuzhilin, R. D. Burke, A. Felfernig, and L. Schmidt-Thieme, editors, RecSys, pages 237-240. ACM, 2009.

17. T. Pedersen, S. Patwardhan, and J. Michelizzi. Wordnet::similarity: measuring the relatedness of concepts. In Demonstration Papers at HLT-NAACL 2004, HLTNAACL-Demonstrations '04, pages 38-41, Stroudsburg, PA, USA, 2004. Association for Computational Linguistics.

18. J. Peng and D. Zeng. Exploring information hidden in tags: A subject-based item recommendation approach. In Proceedings of nineteenth Annual Workshop on Information Technologies and Systems (WITS 2009), Phoenix, Arizona, USA, 2009.

19. J. Peng and D. Zeng. Topic-based web page recommendation using tags. In ISI, pages 269-271. IEEE, 2009.

20. J. Peng and D. Zeng. Making item predictions through tag recommendations. In Proceedings of the 2010 International Conference on Electronic-Business Intelligence, ICEBI '10, 2010.

21. J. Peng, D. D. Zeng, H. Zhao, and F.-y. Wang. Collaborative filtering in social tagging systems based on joint item-tag recommendations. In Proceedings of the 19th ACM international conference on Information and knowledge management, CIKM '10, pages 809-818, New York, NY, USA, 2010. ACM.

22. G. Pitsilis, X.-L. Zhang, and W. Wang. Clustering recommenders in collaborative filtering using explicit trust information. In I. Wakeman, E. Gudes, C. D. Jensen, and J. Crampton, editors, Trust Management V, 5th IFIP WG 11.11 International Conference, IFIPTM 2011, Copenhagen, Denmark, June 29-July 1, 2011, Proceedings, volume 358 of IFIP Publications, pages 82-97. Springer, 2011.

23. P. Resnik. Using information content to evaluate semantic similarity in a taxonomy. In Proceedings of the 14th international joint conference on Artificial intelligence - Volume 1, IJCAI'95, pages 448-453, San Francisco, CA, USA, 1995. Morgan Kaufmann Publishers Inc.

24. G. Salton, A. Wong, and C. S. Yang. A vector space model for automatic indexing. Commun. ACM, 18(11):613-620, Nov. 1975.

25. A. Shepitsen, J. Gemmell, B. Mobasher, and R. Burke. Personalized recommendation in social tagging systems using hierarchical clustering. In Proceedings of the 2008 ACM conference on Recommender systems, RecSys '08, pages 259-266, New York, NY, USA, 2008. ACM.

26. X.-F. Su, H.-J. Zeng, and Z. Chen. Finding group shilling in recommendation system. In Special interest tracks and posters of the 14 th international conference on World Wide Web, WWW '05, pages 960-961, New York, NY, USA, 2005. ACM.

27. P. Symeonidis, A. Nanopoulos, and Y. Manolopoulos. Tag recommendations based on tensor dimensionality reduction. In Proceedings of the 2008 ACM conference on Recommender systems, RecSys '08, pages 43-50, New York, NY, USA, 2008. ACM.

28. K. H. L. Tso-sutter, R. B. Marinho, and L. Schmidt-thieme. Tag-aware recommender systems by fusion of collaborative filtering algorithms. In In Proceedings of the 2nd ACM Symposium on Applied Computing. ACM, 1995.

29. P. Venetis, G. Koutrika, and H. Garcia-Molina. On the selection of tags for tag clouds. In Fourth ACM International Conference on Web Search and Data Mining (WSDM2011). Stanford InfoLab, February 2011. 
30. W. Wang, X. Zhang, and G. Pitsilis. Abstracting audit data for lightweight intrusion detection. In Proceedings of the 6th International Conference on Information Systems Security, ICISS'10, pages 201-215, Berlin, Heidelberg, 2010. SpringerVerlag.

31. C. Wei, W. Hsu, and M. L. Lee. A unified framework for recommendations based on quaternary semantic analysis. In Proceedings of the 34 th international ACM SIGIR conference on Research and development in Information Retrieval, SIGIR '11, pages 1023-1032, New York, NY, USA, 2011. ACM.

32. G. Xu, Y. Gu, Y. Zhang, Z. Yang, and M. Kitsuregawa. Toast: a topic-oriented tagbased recommender system. In Proceedings of the 12th international conference on Web information system engineering, WISE'11, pages 158-171, Berlin, Heidelberg, 2011. Springer-Verlag.

33. D. Zeng and H. Li. How useful are tags? - an empirical analysis of collaborative tagging for web page recommendation. In C. C. Yang, H. Chen, M. Chau, K. Chang, S.-D. Lang, P. S. Chen, R. Hsieh, D. Zeng, F.-Y. Wang, K. M. Carley, W. Mao, and J. Zhan, editors, Intelligence and Security Informatics, IEEE ISI 2008 International Workshops: PAISI, PACCF, and SOCO 2008, Taipei, Taiwan, June 17, 2008. Proceedings, volume 5075 of Lecture Notes in Computer Science, pages 320-330. Springer, 2008.

34. X.-L. Zhang, T. Lee, and G. Pitsilis. Securing recommender systems against shilling attacks using social-based clustering. Journal of Computer Science and Technology, 28(4):616-624, 2013. 